\title{
Application of a diazepam milligram equivalency algorithm to assess benzodiazepine dose intensity in Rhode Island in 2018
}

\author{
Eric P Borrelli, PhD, PharmD, MBA; Jeffrey Bratberg, PharmD, FAPhA; Benjamin D Hallowell, PhD; \\ Mary L Greaney, PhD, MPH; and Stephen J Kogut, PhD, MBA, RPh
}

\section{What is already known about this subject}

- Benzodiazepines are indicated for the treatment of many conditions, including anxiety disorders, muscle spasms, alcohol withdrawal, agitation, movement disorders, and epilepsy.

- Greater benzodiazepine dose intensity has been associated with adverse outcomes, including amnesia, somnolence, falls, mortality, as well as an increase in health care resource utilization.

\author{
What this study adds \\ - A standardized diazepam milligram \\ equivalency (DME) algorithm was \\ developed, taking into consideration \\ medication half-lives and daily \\ dosing recommendations to assess \\ benzodiazepine dose intensity. \\ - More than a quarter of patients who \\ received a benzodiazepine prescription \\ in Rhode Island in 2018 (26.2\%) had a \\ daily dose intensity of at least 15 DME \\ per day. \\ - Patients with concurrent use of \\ prescription benzodiazepines and either \\ prescription opioids or prescription \\ stimulants had significantly higher \\ odds of receiving a daily DME of at \\ least $15(\mathrm{aOR}=1.67,95 \% \mathrm{Cl}=1.61$ - \\ 1.72; $\mathrm{aOR}=1.84,95 \% \mathrm{Cl}=1.76-1.93$, \\ respectively).
}

OBJECTIVE: To develop and apply a standardized benzodiazepine milligram equivalency conversion algorithm and assess the dose intensity of benzodiazepine use in Rhode Island (RI) in 2018.

METHODS: A systematic literature review was conducted to identify the most commonly used benzodiazepine equivalency values. We then conducted a cross-sectional analysis of 2018 data from the RI Prescription Drug Monitoring Program (PDMP) to calculate the mean daily diazepam milligram equivalency (DME) based on a patient's most recent dispensing. A multivariable logistic regression analysis was conducted to determine the association between higher benzodiazepine doses ( $\geq 15 \mathrm{DME} /$ day) and recipient

\section{Author affiliations}

Eric P Borrelli, PhD, PharmD, MBA; Jeffrey Bratberg, PharmD, FAPhA; and Stephen J Kogut, PhD, MBA, RPh, University of Rhode Island College of Pharmacy, Kingston. Benjamin D Hallowell, PhD, Rhode Island Department of Health, Providence. Mary L Greaney, PhD, MPH, University of Rhode Island College of Health Sciences, Kingston.

\section{AUTHOR CORRESPONDENCE:}

Eric P Borrelli, EricBorrelli@my.uri.edu

J Manag Care Spec Pharm 2022;28(1):58-68

Copyright $@ 2022$, Academy of Managed Care Pharmacy. All rights reserved.

characteristics, including concurrent use of opioids or stimulants.

RESULTS: We identified 143,026 patients who received at least 1 prescription for a benzodiazepine in RI in 2018. The mean (SD) daily DME was 10.60 (9.05), and $26.2 \%$ of individuals had a mean DME per day of at least 15 . Approximately $14 \%(n=20,168)$ of patients prescribed a benzodiazepine had concurrent use with a prescription opioid, and $6.7 \%$ $(n=9,547)$ had concurrent use with a prescription stimulant. Females had a $28 \%$ lower adjusted odds of receiving a benzodiazepine dose of at least 15 DME per day compared with males (adjusted odds ratio $[\mathrm{aOR}]=0.72$, $95 \% \mathrm{Cl}=0.70-0.73$ ). The adjusted odds of receiving a benzodiazepine prescription of 
at least 15 DME per day was lower among the younger (aged 18-34 years) and older age groups (aged 65 years and older) compared with patients aged 35-64 years. Compared with commercial insurance, all other forms of payment had significantly higher adjusted odds of a daily benzodiazepine dose of at least 15 DME per day. The adjusted odds receiving a daily DME of at least 15 was $67 \%$ higher among those who also received a concurrent pharmacy dispensing for an opioid and $84 \%$ higher among those who also received a concurrent dispensing for a stimulant drug $(\mathrm{aOR}=1.67,95 \% \mathrm{Cl}=1.61-1.72$; $\mathrm{aOR}=1.84,95 \% \mathrm{Cl}=1.76-1.93$, respectively).

CONCLUSIONS: Individuals aged 35-64 years with Medicaid insurance and those aged under 65 years with Medicare were more likely to be prescribed a benzodiazepine of at least 15 DME per day. Higher benzodiazepine DMEs were also dispensed to patients who concurrently used prescription opioids or stimulants who may be at increased risk of medication-related harm. We advocate for routine measurement of benzodiazepine dose intensity as a risk reduction strategy.

Benzodiazepines are indicated for the treatment of many conditions, including anxiety disorders, muscle spasms, alcohol withdrawal, agitation, movement disorders, and epilepsy. ${ }^{1}$ Benzodiazepines are one of the most frequently prescribed medication classes, and 3 benzodiazepines (alprazolam, clonazepam, and lorazepam) occupied positions in the top 60 prescribed drugs in 2020 in the United States. ${ }^{2}$ Data from the 2019 National Survey on Drug Use and Health indicate that approximately 28.4 million or $11.4 \%$ of US adults reported using prescription benzodiazepines during the previous year. ${ }^{3}$

Despite being a commonly prescribed class of medication, benzodiazepines have substantial risk for addiction and are classified as Schedule IV controlled substances by the US Drug Enforcement Administration. Benzodiazepines also increase the risk of falls, leading to fractures and hospitalizations ${ }^{4,5}$; cause memory impairment (eg, amnesia, dementia, and other cognitive declines); and have other central nervous system effects. ${ }^{6}$ When taken concomitantly with opioids, benzodiazepines increase the risk of respiratory depression and opioid overdose, and both classes of medications have a black box warning related to their concurrent use in their prescribing information. ${ }^{7-9}$ While the use of opioids and benzodiazepines concurrently has been decreasing in recent years, ${ }^{10}$ as of 2017, an estimated 1.9 million US patients were still taking benzodiazepines and opioids concurrently. ${ }^{10}$

Benzodiazepines also have specific risks when prescribed for older adults because of the increased sensitivity to and decreased metabolism of these medications associated with aging. ${ }^{11}$ Benzodiazepines are included in the Beers Criteria for Potentially Inappropriate Medication Use in Older Adults, ${ }^{11}$ and the American Psychiatric Association has stated that "benzodiazepine use should be avoided whenever possible" in patients aged 65 years and older. ${ }^{12}$

Higher benzodiazepine dose intensity has been associated with adverse outcomes, including amnesia, somnolence, falls, mortality, and increased health care resource utilization (eg, emergency department visits and hospital admissions). ${ }^{13-16}$ Measuring benzodiazepine dose intensity in a manner similar to how daily morphine milligram equivalents (MME) for opioids are measured may promote safer use of benzodiazepines at the patient and population levels.

The aim of this study was to develop and apply a standardized benzodiazepine milligram equivalency conversion algorithm to assess the dosing intensity of benzodiazepine prescriptions dispensed in Rhode Island (RI) in 2018. We also sought to determine if subgroups at an increased risk of experiencing medication-related harm were receiving higher doses of benzodiazepines when compared with other groups.

\section{Methods}

This study had 2 parts: developing a benzodiazepine equivalency algorithm and then applying the equivalency algorithm to data from the RI Prescription Drug Monitoring Program (PDMP). To develop the benzodiazepine equivalency algorithm, a literature review was conducted to identify the most commonly used benzodiazepine equivalency values. Only articles that created a benzodiazepine equivalency dose were included. Articles that established an equivalency dose based on serum concentrations or parenteral dosages were excluded, since an equivalency dose is based on blood levels and does not account for oral medications undergoing drug metabolism. All identified abstracts were reviewed for inclusion. Abstracts that met the inclusion criteria had their full texts reviewed. Our scan included a search of relevant articles in PubMed/Medline, Google, and a review of citations. The search included the query terms "benzodiazepine" AND "equivalents" and yielded 205 studies.

After excluding studies that did not meet our inclusion criteria, 16 remaining studies were examined to identify the most commonly used equivalency for each benzodiazepine type (Supplementary Table 1, available in online article). ${ }^{17-32}$ The recommended total daily dose described in product labels was used to adjust for differences in dosing intervals and to standardize total daily dose exposure across medication types to create a diazepam milligram equivalency (DME). ${ }^{33-44}$ For example, clonazepam has an approved daily 


\section{TABLE 1 Diazepam Milligram Equivalency Algorithm}

\begin{tabular}{l|c|c}
\hline \multicolumn{1}{c|}{ Medication } & Equivalence & Conversion factor \\
\hline Alprazolam & $0.50 \mathrm{mg}$ & 10.00 \\
\hline Chlordiazepoxide & $12.50 \mathrm{mg}$ & 0.40 \\
\hline Clobazam & $10.00 \mathrm{mg}$ & 0.50 \\
\hline Clonazepam & $0.50 \mathrm{mg}$ & 10.00 \\
\hline Clorazepate & $7.50 \mathrm{mg}$ & 0.67 \\
\hline Diazepam & $5.00 \mathrm{mg}$ & 1.00 \\
\hline Estazolam & $0.67 \mathrm{mg}$ & 7.50 \\
\hline Flurazepam & $15.00 \mathrm{mg}$ & 0.33 \\
\hline Lorazepam & $1.00 \mathrm{mg}$ & 5.00 \\
\hline Oxazepam & $15.00 \mathrm{mg}$ & 0.33 \\
\hline Temazepam & $10.00 \mathrm{mg}$ & 0.50 \\
\hline Triazolam & $0.25 \mathrm{mg}$ & 20.00
\end{tabular}

dose ranging from 0.5 cumulative milligrams to 4 cumulative milligrams per day (excluding seizure disorder), ${ }^{36}$ whereas diazepam has an approved daily dose ranging from 2 to 40 cumulative daily milligrams. ${ }^{38}$ The maximum daily approved dosage of diazepam is 10 times that of clonazepam, which equates to a daily equivalency of $0.5 \mathrm{mg}$ for clonazepam compared with $5 \mathrm{mg}$ of diazepam, which is a 10 -fold difference (Table 1). DME was thus calculated as the ([dose conversion factor $] \times[$ the medication strength $] \times[$ the prescription quantity]) $\div$ (number of days supply dispensed).

To implement the developed equivalency algorithm, we conducted a cross-sectional analysis of data from the RI PDMP for the 2018 calendar year, subsequent to obtaining a data use agreement with the RI Department of Health. The RI PDMP database includes prescription controlled substances dispensed by all retail pharmacies in RI with a controlled substance registration. In addition, the RI PDMP database includes controlled substances dispensed to RI residents by pharmacies in all New England states and 32 states in total. ${ }^{45}$

Individuals with at least 1 dispensing of a benzodiazepine during 2018 were included in the analytic sample. Patients were excluded if they were aged under 18 years or were prescribed opioid formulations used primarily for patients diagnosed with cancer or were enrolled in hospice care, since this may require higher benzodiazepine doses (Figure 1).

Prescription claims were excluded if they were veterinarian prescriptions, missing drug identifiers, or were for gel diazepam or injectable midazolam, since these dosage forms are generally not used by outpatients on a daily basis. Drug powder formulations were excluded from the analysis because we were unable to accurately determine the correct daily dose. Payment status of "commercial insurance" for patients aged 65 years and older was reclassified as "Medicare," since it appeared that Medicare Advantage plans were often misclassified in the dataset as commercial insurance.

The dependent variable for the analysis was dosages of benzodiazepines calculated according to the most recent dispensing received in 2018, which was dichotomized for all analyses. We did not locate any consensus thresholds that specifically defined higher or lower benzodiazepine doses, and published papers on this topic have used a variety of thresholds and methods..$^{13-16,46-49}$ The threshold of at least 15 DME per day was chosen for this analysis because it was a $50 \%$ higher dose than the median DME per day of 10 and also represented the upper value of the interquartile range. Increased risk of adverse outcomes have been seen with increases in benzodiazepine dose ${ }^{13-16,46-49}$ for example, doses greater than 10 through 20 DME per day have a higher risk of opioid overdose when concurrently taken with opioids compared with doses of 10 DME per day or less. ${ }^{47}$

Demographic covariates included sex, age group, payment method, and RI county of residence. We also identified individuals who had at least 7 days of overlap between any benzodiazepine prescription during 2018 and a dispensing for a prescription opioid or attention deficit hyperactivity disorder stimulant drug. ${ }^{50-52}$

Descriptive statistics were used to present the frequency and percentage of patients receiving a benzodiazepine prescription at a dose of at least 15 DME per day, overall and for each subgroup. A multivariable logistic regression analysis was performed to determine the association between patient characteristics and use of benzodiazepines at a DME per day of at least 15 (yes or no). Independent variables with a $P$ value of less than 0.25 in bivariate analyses were included in the final model..$^{53,54}$ Collinearity was assessed by examining condition indices ${ }^{55,56}$; however, no independent variables were deemed to be collinear. Therefore, all variables were included in the analysis. The measure of effect was the adjusted odds ratio (aOR) and corresponding 95\% $\mathrm{CI}$, which represents the odds of receiving a benzodiazepine dispensing with at least 15 DME per day, after adjusting for covariates.

All analyses were performed using SAS version 9.4 (SAS Institute). This study was approved by the institutional review boards at the RI Department of Health and at the University of Rhode Island. 


\section{FIGURE 1 Sample Identification Flowchart}

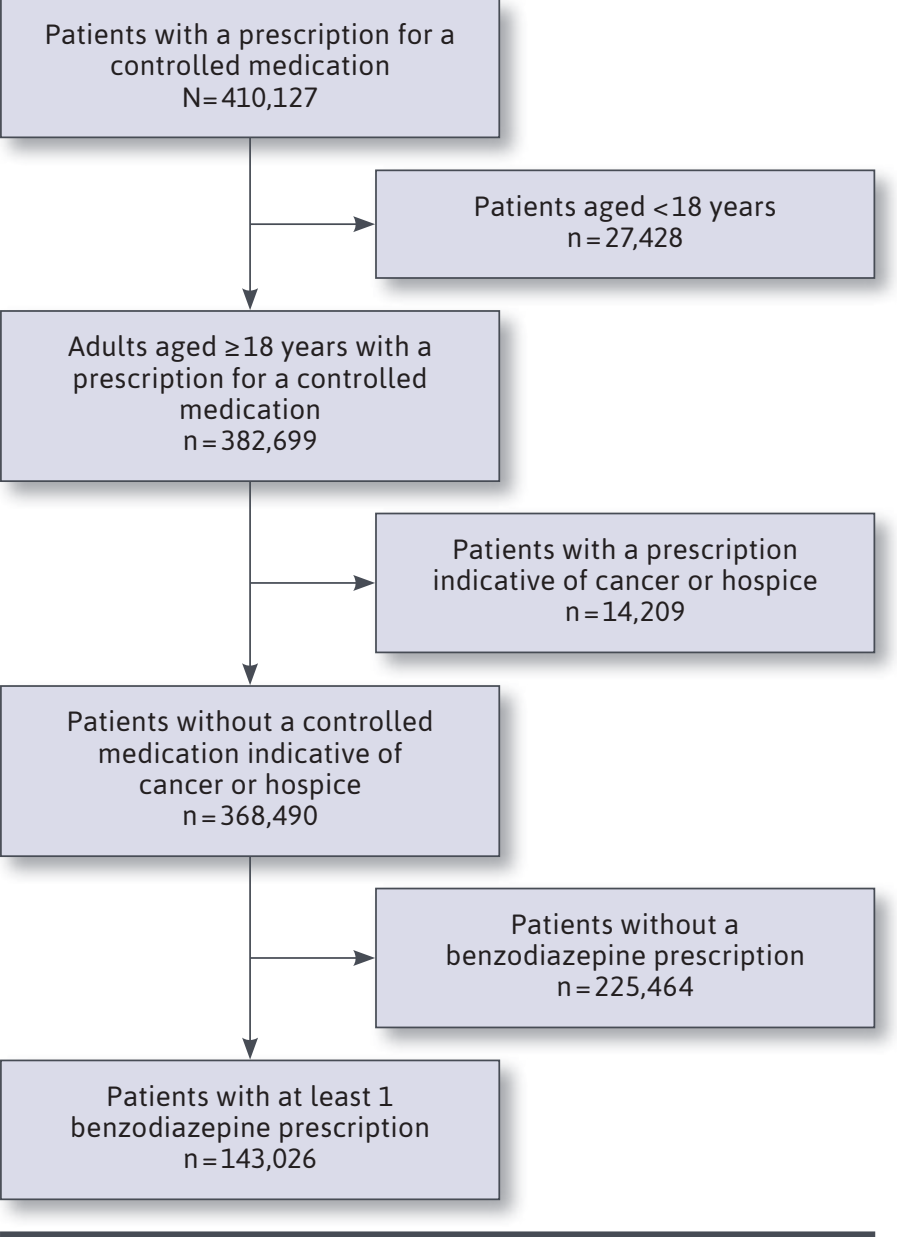

\section{Results}

Of the 154,918 patients who were dispensed at least 1 prescription benzodiazepine in 2018, 143,026 were included in the final sample (Table 2). The study population was $66.3 \%$ female $(n=94,884) ; 34.6 \%(n=49,444)$ of patients were aged 50-64 years; and 30.7\% $(n=43,903)$ of patients were aged 65 years and older (Table 3). Nearly half of patients filled their benzodiazepine prescriptions using commercial insurance $(\mathrm{n}=65,835,46.0 \%)$. In addition, $14.1 \%(\mathrm{n}=20,168)$ of patients received a prescription opioid that overlapped with a dispensed benzodiazepine by at least 7 days, whereas $6.7 \%$ $(n=9,547)$ received an overlapping stimulant drug.

Clonazepam, alprazolam, lorazepam, and diazepam comprised more than $95 \%$ of all benzodiazepine prescriptions dispensed at the patient and prescription levels, while also comprising $94.8 \%$ of the included benzodiazepine dispensings having a dose intensity of at least 15 DME per day.

The population's mean (SD) DME per day was 10.60 (9.05) and median DME per day was 10.00 (interquartile range $[\mathrm{IQR}]=5.00-15.00 ; 10 \%-90 \%$ range $=2.50-20.00)$. The median DME per day, IQR, and the 10\%-90\% range were consistent with the recommended dosing of diazepam. More than a quarter of the study population (26.2\%) were dispensed a benzodiazepine prescription of at least 15 DME per day. Although there were almost twice as many females who received prescriptions for benzodiazepines than males (66.3\% to $33.6 \%)$, a higher percentage of males than females received at least 15 DME per day (30.3\% vs $24.1 \%)$.

Among payment types, the proportion of patients who received at least 15 DME per day (according to the most recent dispensing) was similar for commercial insurance, cash payment, and Medicare beneficiaries aged 65 years and older (21.1\%-24.0\%). However, 35.4\% $(n=6,177)$ of those covered by Medicaid and $46.2 \%(n=4,378)$ of those covered by Medicare who were under the age of 65 received a benzodiazepine dispensing with at least 15 DME per day. Patients with overlapping prescriptions for benzodiazepines and stimulants had one of the highest percentages of recent benzodiazepine dispensings with at least 15 DME per day $(39.4 \%, n=3,760)$, whereas among those having overlapping dispensings for opioids, $37.4 \%(n=7,537)$ had recent benzodiazepine dispensings of at least 15 DME per day.

The multivariable analysis identified several statistically significantly different subgroups receiving a benzodiazepine dose of at least 15 DME per day (Table 4). Females were significantly less likely to receive a benzodiazepine dose of at least 15 DME per day than males after adjusting for age group, payment method, region, and concurrent use of opioids or stimulants $(\mathrm{aOR}=0.72,95 \% \mathrm{CI}=0.70-0.73)$. As compared with patients aged 35-49 years, all other age groups (18-34 years, 65-74 years, and $75^{+}$) had lower adjusted odds of a daily benzodiazepine dose of at least 15 DME except for those aged 50-64 years, for which there was no significant difference $(\mathrm{aOR}=1.00,95 \% \mathrm{CI}=0.97-1.04)$.

Furthermore, compared with patients using commercial insurance, patients using other payment methods had a higher adjusted odds of receiving a benzodiazepine dose of at least 15 DME per day. This was highest among Medicare patients aged under 65 years who had nearly 2.5 times the adjusted odds of receiving a recent benzodiazepine dose of at least 15 DME per day, as compared with patients using commercial insurance $(\mathrm{aOR}=2.44,95 \% \mathrm{CI}=2.33-2.56)$.

Patients with concurrent use of benzodiazepines and opioids had $67 \%$ higher adjusted odds of receiving a benzodiazepine dose of at least 15 DME per day $(\mathrm{aOR}=1.67,95 \%$ $\mathrm{CI}=1.61-1.72)$, whereas patients with concurrent use with 


\section{TABLE 2}

\section{Number of Prescriptions per Benzodiazepine Type, Rhode Island Prescription Drug Monitoring Program, 2018}

\begin{tabular}{l|rr|rr}
\hline \multicolumn{1}{|c|}{ Medication } & \multicolumn{2}{|c|}{$\begin{array}{c}\text { Total prescriptions, } \\
\mathbf{n ( \% )}\end{array}$} & $\begin{array}{c}\text { Number (\%) } \\
\text { of patients } \\
\text { (according to the } \\
\text { most recent } \\
\text { dispensing in 2018) }\end{array}$ \\
\hline Clonazepam & 175,224 & $(31.4)$ & $37,048 \quad(25.9)$ \\
\hline Alprazolam & 157,053 & $(28.2)$ & 41,091 & $(28.7)$ \\
\hline Lorazepam & 146,892 & $(26.4)$ & 42,260 & $(29.6)$ \\
\hline Diazepam & 51,607 & $(9.3)$ & 16,575 & $(11.6)$ \\
\hline Temazepam & 18,111 & $(3.2)$ & 3,657 & $(2.6)$ \\
\hline Triazolam & 3,010 & $(0.5)$ & 1,139 & $(0.8)$ \\
\hline Clorazepate & 2,073 & $(0.4)$ & 485 & $(0.3)$ \\
\hline Chlordiazepoxide & 1,493 & $(0.3)$ & 400 & $(0.3)$ \\
\hline Oxazepam & 812 & $(0.1)$ & 182 & $(0.1)$ \\
\hline Clobazam & 556 & $(0.1)$ & 92 & $(0.1)$ \\
\hline Flurazepam & 363 & $(0.1)$ & 79 & $(0.1)$ \\
\hline Estazolam & 77 & $(<0.1)$ & 19 & $(<0.1)$ \\
\hline
\end{tabular}

stimulants had an $84 \%$ higher adjusted odds of receiving a benzodiazepine dose of at least 15 DME per day $(\mathrm{aOR}=1.84$, $95 \% \mathrm{CI}=1.76-1.93)$.

\section{Discussion}

This study demonstrated an approach to identify patient subgroups prescribed high doses of benzodiazepines who may be at greater risk of benzodiazepine-related adverse events. In our analysis, these high-risk subgroups included males, patients aged 35-64 years, those with public insurance (as compared with commercial insurance), and patients who concurrently used benzodiazepines with either opioids or stimulants.

Benzodiazepines are among the most commonly prescribed medications in the United States, notwithstanding their substantial risks, which include addiction, memory impairment, and falls leading to fractures and hospitalizations, particularly among older adults..$^{4-6}$ A meta-analysis of studies published between 1996 and 2007 found that older adults prescribed benzodiazepines had a $57 \%$ higher odds of falling, as compared with patients not prescribed these drugs. ${ }^{57}$ Benzodiazepines are an increasing focus of deprescribing initiatives, given their ubiquity of use and the difficulty that many patients have discontinuing these drugs ${ }^{58}$ While benzodiazepines should not be discontinued abruptly, risks of harm can be decreased by gradually tapering the prescribed regimen toward eventual cessation. Although several benzodiazepine equivalency conversions have been previously developed, ${ }^{17-32}$ none of the identified equivalency algorithms include all 12 oral benzodiazepines. In addition, our study used real-world data from more than 140,000 patients to demonstrate the algorithm's applicability to a large population.

Assessing opioid dose intensity using the standardized MME approach is recommended in the Centers for Disease Control and Prevention (CDC) guidelines for opioid prescribing. ${ }^{59} \mathrm{~A}$ similar approach for benzodiazepines would aid state departments of health, payers, and health care systems to assess and monitor the dose intensity of prescribed benzodiazepines. This would be particularly useful for identifying higher-risk benzodiazepine use among groups such as older adults and those concurrently prescribed other controlled medications. Calculating benzodiazepine dose intensity as DME per day may also help to guide deprescribing., ${ }^{458,60-62}$

Several studies have reported an increase in adverse events when patients use higher doses of benzodiazepines. ${ }^{15,16,46-49}$ An analysis conducted by Kroll et al using data from the Brigham and Women's Primary Care PracticeBased Research Network from July 2011 through June 2012 found that patients who received higher dose benzodiazepines had higher rates of emergency department visits (rate ratio $[\mathrm{RR}]=1.66,95 \% \mathrm{CI}=1.49-1.85$ ) and hospitalizations $(\mathrm{RR}=1.81,95 \% \mathrm{CI}=1.57-2.08)$ compared with those who were prescribed lower doses. ${ }^{16}$ Another US study among hospitalized adults aged 45 years or older found that falls were associated with recent benzodiazepine initiation or dose escalation. ${ }^{48}$

In addition, a case-control analysis was conducted to examine the risks of hospitalization for fall-related injuries in patients aged 65 years and older from the Taiwan National Health Insurance Database..$^{49}$ This analysis found that higher doses of benzodiazepines had a numerically higher aOR of fall-related hospitalizations compared with no benzodiazepine use (low dose aOR $=1.27,95 \% \mathrm{CI}=1.08-$ 1.50; medium dose aOR=1.54, 95\% CI=1.28-1.85; high dose $\mathrm{aOR}=1.75,95 \% \mathrm{CI}=1.47-2.08$ ), although confidence intervals overlapped. ${ }^{49}$

Evaluating benzodiazepine dose intensity is particularly crucial among individuals prescribed opioids or who are diagnosed with opioid use disorder, since higher doses of benzodiazepines increase the risk of overdose when combined with opioids of any MME. A case cohort study by Park et al using data from the US Veterans Health Administration database from 2004 to 2009 of patients prescribed opioids 


\section{TABLE 3}

\begin{tabular}{|c|c|c|c|c|}
\hline \multirow{2}{*}{$\begin{array}{l}\text { Patient demographics } \\
\text { Overall }\end{array}$} & \multicolumn{2}{|c|}{$\begin{array}{l}\text { Patients with a } \\
\text { benzodiazepine } \\
\text { prescription, } \mathrm{n}(\%)\end{array}$} & \multicolumn{2}{|c|}{$\begin{array}{c}\text { Patients with a } \\
\text { benzodiazepine } \\
\text { dose } \geq 15 \\
\text { DME/day, } \mathbf{n}(\%)\end{array}$} \\
\hline & 143,026 & $(100)$ & 37,455 & $(26.2)$ \\
\hline \multicolumn{5}{|l|}{ Sex } \\
\hline Male & 48,106 & $(33.6)$ & 14,556 & $(30.3)$ \\
\hline Female & 94,884 & $(66.3)$ & 22,883 & $(24.1)$ \\
\hline \multicolumn{5}{|l|}{ Age, years } \\
\hline $18-34$ & 18,672 & (13.1) & 4,143 & $(22.2)$ \\
\hline $35-49$ & 31,007 & $(21.7)$ & 9,027 & $(29.1)$ \\
\hline $50-64$ & 49,444 & (34.6) & 14,401 & (29.1) \\
\hline $65-74$ & 25,745 & $(18.0)$ & 6,651 & $(25.8)$ \\
\hline $75+$ & 18,158 & $(12.7)$ & 3,233 & $(17.8)$ \\
\hline \multicolumn{5}{|l|}{ Payment } \\
\hline Commercial insurance & 65,835 & $(46.0)$ & 15,105 & $(22.9)$ \\
\hline Medicaid & 17,433 & $(12.2)$ & 6,177 & $(35.4)$ \\
\hline Medicare $<65$ & 9,478 & $(6.6)$ & 4,378 & $(46.2)$ \\
\hline Medicare 65+ & 30,635 & $(21.4)$ & 7,342 & $(24.0)$ \\
\hline Cash & 15,203 & $(10.6)$ & 3,209 & $(21.1)$ \\
\hline Other & 4,442 & $(3.2)$ & 1,244 & $(28.0)$ \\
\hline \multicolumn{5}{|l|}{ County } \\
\hline Providence & 67,293 & $(47.0)$ & 18,939 & $(28.1)$ \\
\hline Kent & 24,844 & $(17.4)$ & 7,005 & $(28.2)$ \\
\hline Washington & 15,383 & $(10.8)$ & 3,805 & $(24.7)$ \\
\hline Bristol & 5,775 & $(4.0)$ & 1,375 & $(23.8)$ \\
\hline Newport & 8,825 & $(6.2)$ & 2,060 & $(23.3)$ \\
\hline Out of state & 20,906 & $(14.6)$ & 4,271 & $(20.4)$ \\
\hline \multicolumn{5}{|l|}{ Concurrent opioid use } \\
\hline Yes & 20,168 & $(14.1)$ & 7,537 & $(37.4)$ \\
\hline No & 122,858 & $(85.9)$ & 29,918 & $(24.4)$ \\
\hline \multicolumn{5}{|l|}{ Concurrent stimulant use } \\
\hline Stimulant & 9,547 & $(6.7)$ & 3,760 & $(39.4)$ \\
\hline No stimulant & 133,478 & $(93.3)$ & 33,695 & $(25.2)$ \\
\hline \multicolumn{5}{|c|}{$\begin{array}{l}{ }^{a} \text { According to the patient's most recent benzodiazepine dispensing. } \\
\text { bSex was missing for } 36 \text { patients. } \\
\text { DME = diazepam milligram equivalents. }\end{array}$} \\
\hline
\end{tabular}

for acute or chronic nonterminal pain found that recent increases in benzodiazepine dose significantly increased the risk of opioid overdose death, after adjusting for patient covariates. ${ }^{47}$ Moreover, approximately 1 in 3 prescription opioid overdose deaths reported to the CDC between 1999 and 2017 involved benzodiazepines. ${ }^{63}$

We also found that $6.7 \%$ of patients who received benzodiazepines also concurrently received prescription stimulants, and those using stimulants had $84 \%$ higher odds of receiving a high-dose benzodiazepine $(\mathrm{aOR}=1.84$, $95 \% \mathrm{CI}=1.76-1.93)$. We were unable to identify any published study assessing benzodiazepine dose intensity among patients who concurrently received prescription stimulants. While this combination is not contraindicated, both medication classes have a high risk of physical and psychological dependence, and their opposing central nervous system effects may increase the risk of tolerance, abuse, and overdose. ${ }^{64}$ Monitoring benzodiazepine dose intensity may help to mitigate these risks.

The use of higher-dose benzodiazepines was associated with age. As compared with patients aged 35-49 years, those aged 75 years and older had a $58 \%$ lower adjusted odds of receiving a benzodiazepine with a daily DME of at least $15 \mathrm{mg}(\mathrm{aOR}=0.42,95 \% \mathrm{CI}=0.39-0.45)$, and among those aged $65-74$ years, the risk was $35 \%$ lower $(\mathrm{aOR}=0.65,95 \%$ $\mathrm{CI}=0.60-0.70$ ). These results suggest that prescribers may have recognized the potential risks of higher use in older adults and therefore prescribed lower doses. However, despite the less frequent use of higher-dose benzodiazepines among older patients in our study, patients aged 65 years and older represented $30.7 \%$ of the study population of all benzodiazepine users.

The frequent use of benzodiazepines among older adults in our study is highly concerning. Benzodiazepines have been associated with increased risk of cognitive impairment, delirium, falls, fractures, and motor vehicle crashes among older adults and are included in the Beers Criteria for Potentially Inappropriate Medication Use in Older Adults. ${ }^{11}$ Moreover, benzodiazepine dose intensity has been associated with adverse outcomes in older patients. ${ }^{14,65,66}$ Given the increased susceptibility of older adults to the harms of benzodiazepines, thresholds lower than $15 \mathrm{DME}$ per day may be more suitable if these medications must be used.

Our study also identified differences in the use of higher-dose benzodiazepines by patient sex. Approximately twice as many females than males received benzodiazepines (94,884 vs 48,106), yet females were less likely to receive a benzodiazepine dispensing at a DME per day of $15 \mathrm{mg}$ or greater $(\mathrm{aOR}=0.72,95 \% \mathrm{CI}=0.70-0.73)$. These findings are consistent with what has been reported in the literature. ${ }^{16,67-69}$ 


\section{TABLE 4 Adjusted Odds of Receiving At Least 15 DME per Day According to Demographic Covariates and Concurrent Use of Opioids or Stimulants}

\begin{tabular}{|c|c|c|}
\hline Patient demographics & aOR & $95 \% \mathrm{Cl}$ \\
\hline \multicolumn{3}{|l|}{ Sex } \\
\hline Male & \multicolumn{2}{|c|}{ Reference } \\
\hline Female & 0.72 & $0.70-0.73$ \\
\hline \multicolumn{3}{|l|}{ Age, years } \\
\hline $18-34$ & 0.73 & $0.70-0.76$ \\
\hline $35-49$ & \multicolumn{2}{|c|}{ Reference } \\
\hline $50-64$ & 1.00 & $0.97-1.04$ \\
\hline $65-74$ & 0.65 & $0.60-0.70$ \\
\hline $75+$ & 0.42 & $0.39-0.45$ \\
\hline \multicolumn{3}{|l|}{ Payment } \\
\hline Commercial insurance & \multicolumn{2}{|c|}{ Reference } \\
\hline Medicaid & 1.83 & $1.76-1.90$ \\
\hline Medicare < 65 & 2.53 & 2.42-2.65 \\
\hline Medicare 65+ & 1.82 & $1.70-1.96$ \\
\hline Cash & 1.30 & $1.23-1.38$ \\
\hline Other & 1.60 & $1.48-1.73$ \\
\hline \multicolumn{3}{|l|}{ County } \\
\hline Providence & \multicolumn{2}{|c|}{ Reference } \\
\hline Kent & 1.01 & $0.98-1.05$ \\
\hline Washington & 0.88 & $0.84-0.92$ \\
\hline Bristol & 0.84 & $0.79-0.90$ \\
\hline Newport & 0.78 & $0.74-0.82$ \\
\hline Out of state & 0.72 & $0.69-0.74$ \\
\hline \multicolumn{3}{|l|}{ Concurrent opioid use } \\
\hline Yes & 1.67 & $1.61-1.72$ \\
\hline No & \multicolumn{2}{|c|}{ Reference } \\
\hline \multicolumn{3}{|l|}{ Concurrent stimulant use } \\
\hline Yes & 1.84 & $1.76-1.93$ \\
\hline No & \multicolumn{2}{|c|}{ Reference } \\
\hline $\begin{array}{l}\text { Note: Statistically significant } \\
a O R=\text { adjusted odds ratio; } D M\end{array}$ & $\begin{array}{l}\text { bold. } \\
\text { n milligr }\end{array}$ & ents. \\
\hline
\end{tabular}

A study conducted of outpatient prescription claims in the Drug Program Information Network in Canada from April 2001 through March 2016 found that males aged 18-64 years and males aged 65 years and older received benzodiazepines at a higher mean DME per day than females of the same age groups. ${ }^{69}$ Kroll et al found that females had substantially lower odds of receiving benzodiazepines at doses of at least $30 \mathrm{DME}$ per day than males $(\mathrm{OR}=0.62,95 \%$ $\mathrm{CI}=0.52-0.75){ }^{16}$ This finding is difficult to explain, since prescribing recommendations do not specify different dosing according to sex or weight. One possible explanation for this difference might be if the indications for use differed substantially between males and females, which we could not determine from this dataset.

Approximately $35 \%$ of patients using Medicaid as a payment source and $46 \%$ of patients aged under 65 years who used Medicare as a payment source received a benzodiazepine prescription with a dose of at least 15 DME per day, as compared with only $23 \%$ of patients with commercial insurance. The use of higher doses of benzodiazepines among patients in public insurance programs has been observed in other studies. ${ }^{16}$ Kroll et al found that prescriptions filled through Medicare and Medicaid were associated with a higher odds of high dose benzodiazepine use ( $\geq 30 \mathrm{DME} /$ day) compared with private insurance $(\mathrm{aOR}=1.36,95 \% \mathrm{CI}=1.10-1.68 ; \mathrm{aOR}=3.57,95 \% \mathrm{CI}=2.78-4.58$, respectively). ${ }^{16}$ The difference in use of higher-dose benzodiazepines between commercial and public payers may be due to differences in the characteristics of enrollees, rather than differences in policies or effectiveness in utilization management. For example, patients with more chronic or severe mental health conditions may be treated with higher doses of benzodiazepines, and these conditions are more prevalent in Medicaid and Medicare than with commercial insurance.

In 2016, Medicaid covered approximately only $14 \%$ of US adults but covered $21 \%$ of adults with mental illnesses and $17 \%$ of adults with substance use disorder, which may have led to the differences seen in benzodiazepine dose intensity between the different payment types. ${ }^{70}$ In addition, even with a smaller covered population, Medicaid patients made up a larger percentage of inpatient (10\% vs $4 \%$ ) and outpatient visits (49\% vs $42 \%$ ) of all serious mental illnesses among nonelderly US adults in 2015 compared with patients with private insurance. ${ }^{71}$ While we were not able to assess socioeconomic status or social determinants from the RI PDMP, a study examining US Medicaid state drug utilization data for 2010-2017 found that household income, poverty rate, unemployment rate, and Medicaid expansion status were not associated with benzodiazepine dose intensity. ${ }^{72}$

We also found that patients who concomitantly received opioids during the study period had a $67 \%$ higher adjusted odds of receiving a benzodiazepine dose of at least 15 DME per day, as compared with those who did not receive concurrent opioids $(\mathrm{aOR}=1.67,95 \% \mathrm{CI}=1.61-1.72)$. As more attention has been brought to the risks of concurrent use of opioids and benzodiazepines, the prevalence of 
concurrent use has decreased over the last few years following the US Food and Drug Administration alert and black box warning additions. ${ }^{10,73}$ In addition, naloxone coprescribing has significantly increased among patients with concurrent use of opioids and benzodiazepines. With this in mind, we expected that benzodiazepine dosing would be lower among those concurrently using opioids, given the increased risk of overdose with this combination. However, our findings were the opposite. We surmise that these patients with pain required increasing doses of benzodiazepines with continued use. Both opioids and benzodiazepines have black box warnings that describe the risk of overdose associated with concomitant use.,974

The previously mentioned study by Park et al found an increasing risk of death from drug overdose with increasing the DME per day. ${ }^{47}$ Compared with patients receiving a daily DME of greater than 0 to $10 \mathrm{mg}$, the adjusted hazards ratio (aHR) for death from drug overdose increased from 1.69 (95\% CI =1.42-2.01) for patients receiving more than $10-20$ DME per day to an aHR of 3.06 (95\% $\mathrm{CI}=2.38-3.92$ ) for patients who had a daily DME exceeding $40 \mathrm{mg}$ per day. ${ }^{47}$

\section{LIMITATIONS}

This study's findings should be considered with respect to its limitations. The dataset did not include diagnosis codes, so we could not determine which clinical diagnoses corresponded with benzodiazepine dose intensity. Other information, such as comorbid conditions, prescriber type and specialty, and health care resource utilization, are examples of potentially important covariates that we were not able to assess. In addition, we were unable to determine the degree to which clinical indication or duration of use was related to dose intensity.
Also, we were not able to account for patient race or ethnicity, socioeconomic factors, or social determinants of health. The DME algorithm was limited in that it did not take into account differing metabolism effects, since certain benzodiazepines, such as lorazepam, are recommended to have dose reductions in patients with liver dysfunction. ${ }^{41}$ The RI PDMP does not include prescription directions, so calculated dose intensity relies on the correct input of the days supply by pharmacy staff. However, during data cleaning we did remove prescriptions having a quantity per day with an illogical value that indicated a high likelihood of transcription error, which helped remove some instances of incorrect input of days supply.

These limitations are counterbalanced by several strengths, which include the data source, the precision with which we measured benzodiazepine dose intensity, and our evaluation of high-dose benzodiazepine use among important subgroups.

\section{Conclusions}

This study used a DME per day approach to identify higher-dose benzodiazepine use among different population subgroups using the RI PDMP dataset. Males, patients with Medicaid insurance, patients with Medicare aged less than 65 years, and patients aged 35-64 years were subgroups that more frequently received higher doses of benzodiazepines. Patients who concurrently received benzodiazepines with opioids or stimulants also had higher odds of receiving benzodiazepine doses of at least 15 DME per day. These groups are at increased risk of experiencing medication-related harm. We advocate for routine measurement of benzodiazepine dose intensity as a risk reduction strategy.

\section{DISCLOSURES}

No funding supported this study. The authors have no conflicts of interest to disclose. The content and results of this study are solely the responsibility of the authors and do not necessarily represent the official views of the Rhode Island Department of Health. Kogut is partially supported by Institutional Development Award Numbers U54GM115677 and P20GM125507 from the National Institute of General Medical Sciences of the National Institutes of Health, which funds Advance Clinical and Translational Research (Advance-CTR) and the RI Lifespan Center of Biomedical Research Excellence (COBRE) on Opioids and Overdose, respectively. The content of this study is solely the responsibility of the authors and does not necessarily represent the official views of the National Institutes of Health.

Contents of this study were presented as a poster presentation at AMCP 2019 Nexus; October 29-November 1, 2019; National Harbor, MD.

\section{REFERENCES}

1. Sparks A, Cohen A, Albright B, et al. Benzodiazepine and z-drug safety guideline. Kaiser Permanente. January 2019. Accessed August 19, 2020. https:// wa.kaiserpermanente.org/static/pdf/ public/guidelines/benzo-zdrug.pdf

2. ClinCalc. The top 200 drugs of 2020. Accessed January 19, 2021. http://clincalc. com/DrugStats

3. Center for Behavioral Health Statistics and Quality. Results from the 2019 National Survey on Drug Use and Health: detailed tables. Substance Abuse and Mental Health Services Administration, Center for Behavioral Health Statistics and Quality. 2020. Accessed March 26, 2021. https://www.samhsa.gov/data/ report/2019-nsduh-detailed-tables

4. Leclerc BS, Bégin C, Cadieux E, et al. Risk factors for falling among communitydwelling seniors using home-care services: an extended hazards model with time-dependent covariates and multiple events. Chronic Dis Can. 2008;28(4):111-20. 
5. Yu NW, Chen PJ, Tsai HJ, et al. Association of benzodiazepine and Z-drug use with the risk of hospitalisation for fall-related injuries among older people: a nationwide nested case-control study in Taiwan. BMC Geriatr. 2017;17(1):140.

6. Markota M, Rummans TA, Bostwick JM, Lapid MI. Benzodiazepine use in older adults: dangers, management, and alternative therapies. Mayo Clin Proc. 2016;91(11):1632-39.

7. Sun EC, Dixit A, Humphreys K, Darnall BD, Baker LC, Mackey S. Association between concurrent use of prescription opioids and benzodiazepines and overdose: retrospective analysis. BMJ. 2017;356:j760.

8. Jones CM, McAninch JK. Emergency department visits and overdose deaths from combined use of opioids and benzodiazepines. Am J Prev Med. 2015;49(4):493-501.

9. McCarthy M. US drug labels to warn of risks of combining opioids and benzodiazepines. BMJ. 2016;354:i4784.

10. Zhang VS, Olfson M, King M. Opioid and benzodiazepine coprescribing in the United States before and after US Food and Drug Administration boxed warning. JAMA Psychiatry. 2019;76(11):1208-10.

11. The 2019 American Geriatrics Society Beers Criteria® Update Expert Panel. American Geriatrics Society 2019 Updated AGS Beers Criteria® for potentially inappropriate medication use in older adults. J Am Geriatr Soc. 2019;67(4):674-94.

12. Stein MB, Goin MK, Pollack MH, et al. Practice Guideline for the Treatment of Patients with Panic Disorder. 2nd ed. American Psychiatric Association; 2009. Accessed August 18, 2020. http:// www.psychiatryonline.com/pracGuide/ PracticePDFs/PanicDisorder_2e_ PracticeGuideline.pdf.

13. Kothary SP, Brown AC, Pandit UA, Samra SK, Pandit SK. Time course of antirecall effect of diazepam and lorazepam following oral administration. Anesthesiology. 1981;55(6):641-44.
14. Wang PS, Bohn RL, Glynn RJ, Mogun H, Avorn J. Hazardous benzodiazepine regimens in the elderly: effects of half-life, dosage, and duration on risk of hip fracture. Am J Psychiatry. 2001;158(6):892-98.

15. Ekström MP, Bornefalk-Hermansson A, Abernethy AP, Currow DC. Safety of benzodiazepines and opioids in very severe respiratory disease: national prospective study. BMJ. 2014;348:g445.

16. Kroll DS, Nieva HR, Barsky AJ, Linder JA. Benzodiazepines are prescribed more frequently to patients already at risk for benzodiazepine-related adverse events in primary care. J Gen Intern Med. 2016;31(9):1027-34.

17. Alessi-Severini S, Bolton JM, Enns MW, et al. Sustained use of benzodiazepines and escalation to high doses in a Canadian population. Psychiatr Serv. 2016;67(9):1012-18.

18. Soumerai SB, Simoni-Wastila L, Singer C, et al. Lack of relationship between long-term use of benzodiazepines and escalation to high dosages. Psychiatr Serv. 2003;54(7):1006-11.

19. Beg M, Fisher S, Siu D, Rajan S, Troxell L, Liu VX. Treatment of alcohol withdrawal syndrome with and without dexmedetomidine. Perm J. 2016;20(2):49-53.

20. Ng K, Dahri K, Chow I, Legal M. Evaluation of an alcohol withdrawal protocol and a preprinted order set at a tertiary care hospital. Can J Hosp Pharm. 2011;64(6):436-45.

21. Mehdi T. Benzodiazepines revisited. Br J Med Pract. 2012;5(1):501.

22. Taylor D, Patton C, Kapur S. Chapter 4: Depression and anxiety. In: Taylor D, Paton C, Kapur S, eds. The Maudsley Prescribing Guidelines in Psychiatry. 11th ed. Wiley-Blackwell, 2012:197-314.

23. Miller NS, Gold MS. Management of withdrawal syndromes and relapse prevention in drug and alcohol dependence. Am Fam Physician. 1998;58(1):139-46.
24. Vorma H, Naukkarinen H, Sarna S, Kuoppasalmi K. Treatment of out-patients with complicated benzodiazepine dependence: comparison of two approaches. Addiction. 2002;97(7):851-59.

25. Zitman FG, Couvée JE. Chronic benzodiazepine use in general practice patients with depression: an evaluation of controlled treatment and taper-off: report on behalf of the Dutch Chronic Benzodiazepine Working Group. $\mathrm{Br} \mathrm{J}$ Psychiatry. 2001;178:317-24.

26. Ashton $\mathrm{CH}$. Benzodiazepines: how they work and how to withdraw (aka The Ashton Manual). Institute of Neuroscience, Newcastle University. Revised August 2002. Accessed September 13, 2019. https://benzo.org. uk/manual/contents.htm

27. Brett J, Murnion B. Management of benzodiazepine misuse and dependence. Aust Prescr. 2015;38(5):152-55.

28. Ford C, Law F, Barjolin J, Betterton J, Carnwath T. Guidance for the use and reduction of misuse of benzodiazepines and other hypnotics and anxiolytics in general practice. July 2014. Accessed December 5, 2021. https://www.addictionprofessionals.org.uk/Handlers/ Download.ashx?IDMF=8f776bd7-8127485f-8550-b93603ea275f

29. Howard P, Twycross R, Shuster J, Mihalyo M, Wilcock A. Benzodiazepines. J Pain Symptom Manage. 2014;47(5):955-64.

30. Teboul E, Chouinard G. A guide to benzodiazepine selection. Part I: Pharmacological aspects. Can J Psychiatry. 1990;35(8):700-10.

31. Cloos JM. Benzodiazepines and addiction: long-term use and withdrawal (Part 2). Psychiatr Times. 2010;27(8):34-36.

32. Kirkwood CK, Melton ST. Chapter 73: Anxiety disorders I: generalized anxiety, panic, and social anxiety disorders, $p$. In: Dipiro JT, Talbert R, Yee G, et al., eds. Pharmacotherapy: A Pathophysiologic Approach. 7th ed. McGraw-Hill; 2014:1161-78. 
33. Alprazolam. Package insert. Actavis Elizabeth LLC; 2018. Accessed March 13, 2019. https://dailymed.nlm.nih.gov/ dailymed/drugInfo.cfm?setid=a23063c0099a-4256-b95f-3a857bbf704b

34. Chlordiazepoxide. Package insert. Teva Pharmaceuticals USA Inc.; 2016. Accessed March 13, 2019. https://dailymed.nlm.nih.gov/dailymed/drugInfo. cfm?setid=e3ff8641-ae8a-4016-acb1cb1b7890f5ed

35. Clobazam. Package insert. Camber Pharmaceuticals Inc.; 2018. Accessed March 13, 2019. https://dailymed. nlm.nih.gov/dailymed/drugInfo. cfm?setid=cf31b8d1-0d19-4529-99b6$\underline{1 \mathrm{f} 7366064 \mathrm{a} 3 \mathrm{c}}$

36. Clonazepam. Package insert. Accord Healthcare Inc.; 2018. Accessed March 13, 2019. https://dailymed.nlm.nih.gov/ dailymed/drugInfo.cfm?setid=ebc11109e7bf-452d-b675-4b3236d54164

37. Clorazepate. Package insert. Taro Pharmaceuticals USA Inc.; 2019.

Accessed March 13, 2019. https://dailymed.nlm.nih.gov/dailymed/lookup. cfm?setid=499a6989-8912-44b7-blebee8f85ae378f

38. Diazepam. Package insert. Mylan Pharmaceuticals Inc.; 2018. March 13, 2019. https://dailymed.nlm.nih.gov/ dailymed/lookup.cfm?setid=c397a9da862f-4f3f-8109-7d21691de53a

39. Estazolam. Package insert. Actavis Pharma Inc.; 2018. Accessed March 13, 2019. https://dailymed.nlm.nih.gov/ dailymed/drugInfo.cfm?setid=a1e3b4bf22e9-430a-a768-4d86ae886c9e

40. Flurazepam. Package insert. Mylan Pharmaceuticals Inc.; 2018. Accessed March 13, 2019. https://dailymed. nlm.nih.gov/dailymed/drugInfo. cfm?setid $=2$ f2db2f5-49d3-4d47-a08a$628 \mathrm{df} 49 \mathrm{~d} 2120$

41. Lorazepam. Package insert. Actavis Pharma Inc.; 2019. Accessed March 13, 2019. https://dailymed.nlm.nih.gov/ dailymed/lookup.cfm?setid=ad2a0633$\underline{50 f e-4180-b 743-c 1 e 49 f c 110 c 6}$
42. Oxazepam. Package insert. Actavis Pharma Inc.; 2016. Accessed March 13, 2019. https://dailymed.nlm.nih.gov/ dailymed/drugInfo.cfm?setid=a0d5a4c1ec79-42e6-8e8f-ae4d144edb43

43. Temazepam. Package insert. Amneal Pharmaceuticals LLC; 2019. Accessed March 13, 2019. https://dailymed.nlm.nih.gov/dailymed/drugInfo. cfm?setid=be68c6e0-17ad-40fe-9bc158abe24b53f6

44. Triazolam. Package insert. Greenstone LLC; 2019. Accessed March 13, 2019. https://dailymed.nlm.nih.gov/dailymed/ drugInfo.cfm?setid=5add318e-11b9-42f8b052-0d8cebb32fcf

45. Rhode Island Department of Health. Prescription drug monitoring program. Accessed March 8, 2021. https://health. ri.gov/healthcare/medicine/about/ prescriptiondrugmonitoringprogram/

46. Lamson MJ, Sitki-Green D,

Wannarka GL, Mesa M, Andrews P, Pellock J. Pharmacokinetics of diazepam administered intramuscularly by autoinjector versus rectal gel in healthy subjects: a phase I, randomized, open-label, singledose, crossover, singlecentre study. Clin Drug Investig. 2011;31(8):585-97.

47. Park TW, Saitz R, Ganoczy D, Ilgen MA, Bohnert AS. Benzodiazepine prescribing patterns and deaths from drug overdose among US veterans receiving opioid analgesics: case-cohort study. BMJ. 2015;350:h2698.

48. Skinner BW, Johnston EV, Saum LM. Benzodiazepine initiation and dose escalation. Ann Pharmacother. 2017;51(4):281-85.

49. Yu NW, Chen PJ, Tsai HJ, et al. Association of benzodiazepine and Z-drug use with the risk of hospitalisation for fall-related injuries among older people: a nationwide nested case-control study in Taiwan. BMC Geriatr. 2017;17(1):140.

50. Guy GP Jr, Zhang K, Halpin J, Sargent W. An examination of concurrent opioid and benzodiazepine prescribing in 9 states, 2015. Am J Prev Med. 2019;57(5):629-36.
51. Strickler GK, Zhang K, Halpin JF, Bohnert ASB, Baldwin GT, Kreiner PW. Effects of mandatory prescription drug monitoring program (PDMP) use laws on prescriber registration and use and on risky prescribing. Drug Alcohol Depend. 2019;199:1-9.

52. Liu Y, Logan JE, Paulozzi LJ, Zhang K, Jones CM. Potential misuse and inappropriate prescription practices involving opioid analgesics. Am J Manag Care. 2013;19(8):648-58.

53. Stoltzfus JC. Logistic regression: a brief primer. Acad Emerg Med. 2011;18(10):1099-104.

54. Sperandei S. Understanding logistic regression analysis. Biochem Med (Zagreb). 2014;24(1):12-18.

55. Dobson AJ. Chapter 6: Normal linear models. In: Dobson AJ. An Introduction to Generalized Linear Models. 2nd ed. Chapman \& Hall/CRC; 2002:85-114.

56. Kim JH. Multicollinearity and misleading statistical results. Korean J Anesthesiol. 2019;72(6):558-69.

57. Woolcott JC, Richardson KJ, Wiens MO, et al. Meta-analysis of the impact of 9 medication classes on falls in elderly persons. Arch Intern Med. 2009;169(21):1952-60.

58. Ng BJ, Le Couteur DG, Hilmer SN. Deprescribing benzodiazepines in older patients: impact of interventions targeting physicians, pharmacists, and patients. Drugs Aging. 2018;35(6):493-521.

59. Dowell D, Haegerich TM, Chou R. CDC guideline for prescribing opioids for chronic pain - United States, 2016. MMWR Recomm Rep. 2016;65(1):1-49.

60. Pottie K, Thompson W, Davies S, et al. Deprescribing benzodiazepine receptor agonists: evidence-based clinical practice guideline. Can Fam Physician. 2018;64(5):339-51.

61. Reeve E, Ong M, Wu A, Jansen J, Petrovic M, Gnjidic D. A systematic review of interventions to deprescribe benzodiazepines and other hypnotics among older people. Eur J Clin Pharmacol. 2017;73(8):927-35. 
62. Oldenhof E, Anderson-Wurf J, Hall K, Staiger PK. Beyond prescriptions monitoring programs: the importance of having the conversation about benzodiazepine use. J Clin Med. 2019;8(12):2143.

63. Tori ME, Larochelle MR, Naimi TS. Alcohol or benzodiazepine co-involvement with opioid overdose deaths in the United States, 1999-2017. JAMA Netw Open. 2020;3(4):e202361.

64. Lake J, Frydrich V, Gonzales V, Martinez Alonso E, LaFleur J. Concurrent use of benzodiazepines and stimulants. Utah Medicaid DUR Report. July 2017. Accessed February 19, 2021. https://medicaid.utah.gov/pharmacy/ drugutilization/files/Criteria\%20 Review\%20Documents/2017/2017.08\%20 Concurrent\%20stimulants\%20and\%20 benzodiazepines.pdf.

65. Tannenbaum C. Inappropriate benzodiazepine use in elderly patients and its reduction. J Psychiatry Neurosci. 2015;40(3):E27-E28.

66. Billioti de Gage S, Moride Y, Ducruet T, et al. Benzodiazepine use and risk of Alzheimer's disease: case-control study. BMJ. 2014;349:g5205.
67. Olfson M, King M, Schoenbaum M. Benzodiazepine use in the United States. JAMA Psychiatry. 2015;72(2):136-42.

68. Brett J, Maust DT, Bouck Z, et al. Benzodiazepine use in older adults in the United States, Ontario, and Australia from 2010 to 2016. J Am Geriatr Soc. 2018;66(6):1180-85.

69. Brandt J, Alessi-Severini S, Singer A, Leong C. Novel measures of benzodiazepine and Z-drug utilisation trends in a Canadian provincial adult population (2001-2016). J Popul Ther Clin Pharmacol. 2019;26(1):e22-e38.

70. Substance Abuse and Mental Health Services Administration. 2015 National Survey on Drug Use and Health: detailed tables. Center for Behavioral Health Statistics and Quality. 2016. Accessed January 19, 2021. https://www.samhsa. gov/data/sites/default/files/NSDUHDetTabs-2015/NSDUH-DetTabs-2015/ NSDUH-DetTabs-2015.pdf
71. The Henry J Kaiser Family Foundation. Facilitating access to mental health services: a look at Medicaid, private insurance, and the uninsured. June 2017. Accessed March 11, 2021. http://files.kff.org/attachment/ Fact-Sheet-Facilitating-Access-to-MentalHealth-Services-A-Look-at-MedicaidPrivate-Insurance-and-the-Uninsured

72. Liang D, Guo H, Shi Y. Mandatory use of prescription drug monitoring program and benzodiazepine prescribing among U.S. Medicaid enrollees. Subst Abus. 2021;42(3):294-301.

73. Guy GP Jr, Strahan AE, Haegerich T, et al. Concurrent naloxone dispensing among individuals with high-risk opioid prescriptions, USA, 2015-2019. J Gen Intern Med. 2021;36(10):3254-56.

74. US Food and Drug Administration. FDA drug safety communication. FDA warns about serious risks and death when combining opioid pain or cough medicines with benzodiazepines; requires its strongest warning. 2016. Accessed June 23, 2020. https://www.fda.gov/ drugs/drug-safety-and-availability/ fda-drug-safety-communication-fdawarns-about-serious-risks-and-deathwhen-combining-opioid-pain-or 